\title{
THE EXPERIMENTAL FOUNDATIONS OF PARTICLE PHYSICS
}

Second Edition

\author{
ROBERT N. CAHN \\ Lawrence Berkeley National Laboratory \\ GERS ON GOLDHABER \\ Lawrence Berkeley National Laboratory and \\ University of California at Berkeley
}




\section{Contents}

Preface to the Second Edition

page ix

Preface to the First Edition

1 The Atom Completed and a New Particle

2 The Muon and the Pion

3 Strangeness

4 Antibaryons

5 The Resonances

6 Weak Interactions

7 The Neutral Kaon System

8 The Structure of the Nucleon

9 The Jhb, the r, and Charm

10 Quarks, Gluons, and Jets

11 The Fifth Quark

12 From Neutral Currents to Weak Vector Bosons

13 Testing the Standard Model

357

395

14 The Top Quark

416

15 Mixing and CP Violation in Heavy Quark Mesons

434

16 Neutrino Masses and Oscillations

489

17 Epilogue

544

Index

546 